\title{
CASO 20-2015: Actinomicosis pélvica: Relación con Dispositivos Intrauterinos
}

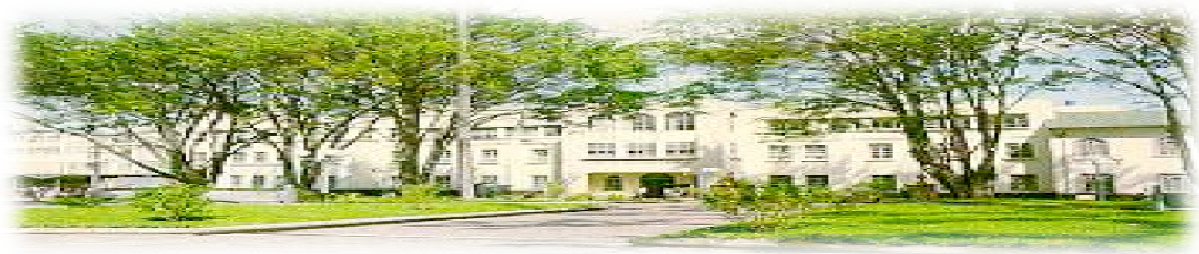

Hospital San Juan de Dios, San José, Costa Rica. Fundado en 1845

\section{ISSN}

Recibido:

$6 / 10 / 2015$

Aceptado:

$17 / 10 / 2015$

Ana Victoria Sánchez Guzmán ${ }^{1}$ Angie Mora Calderón ${ }^{2}$

\begin{abstract}
${ }^{1}$ Médico Asistente General del Servicio de Ginecología del Hospital México. CCSS, San José, Costa Rica. Correo electrónico: anasan75@hotmail.com

${ }^{2}$ Médico Asistente del Servicio de Ginecología del Hospital México. Subespecialista en Ginecología Oncológica.CCSS, San José, Costa Rica. Correo electrónico: angiemora35@hotmail.com
\end{abstract}

\section{RESUMEN}

La infección por actinomyces ha sido llamada "la gran imitadora" en la práctica clínica. Produce un cuadro clínico ambiguo e inespecífico por lo que su diagnóstico es complejo. Su relación con los dispositivos intrauterinos esta ampliamente estudiada.

Esta exposición, de un caso clínico del Servicio de Ginecología del Hospital México, ejemplifica de forma muy acertada la complejidad diagnóstica de esta patología, sus características histológicas y su correlación estrecha con los dispositivos intrauterinos.

\section{PALABRAS CLAVE}

Dispositivos intrauterinos, Actinomyces israelii, infección inflamatoria pélvica, papanicolaou, actinomicosis pélvica

\begin{abstract}
Actinomycosis infection has been called "the great imitator" in the clinical practice. It develops as an ambiguous, nonspecific clinical presentation, which makes the diagnosis difficult. It's relationship with intrauterine devices has been widely studied.

This case report from the Gynecology Department at Mexico Hospital exemplifies very accurately the diagnostic complexity of this disease, its histological characteristics and its close correlation with intrauterine devices.
\end{abstract}

\section{KEY WORDS}

Intrauterine devices, Actinomyces israelii, pelvic inflammatory disease, papanicolaou, pelvic actinomyces. 


\section{CASO CLÍNICO}

Paciente femenina de 49 años, conocida hipertensa crónica en tratamiento con Enalapril $20 \mathrm{mg}$ /día vía oral e Hidroclorotiazida $25 \mathrm{mg}$ /día vía oral. Alérgica a los antiinflamatorios no esteroideos, sin ningún otro antecedente personal no patológico de importancia.

Con múltiples antecedentes quirúrgicos entre los que destacan:

- Apendicectomía a los 14 años.

- Dos Legrados Uterinos Instrumentados por retención placentaria a los 22 años y por aborto retenido a los 24 años respectivamente.

- Resección de masa benigna mamaria derecha a los 40 años.

Entre los antecedentes ginecológicos se documentan 2 gestas, una finalizó en parto vaginal y la segunda en aborto retenido. Fecha de última menstruación 10 días atrás con ciclos menstruales irregulares con patrón de polimenorreas. Planifica con DIU de cobre desde hace 6 años. Con PAP anual sin alteraciones.

Ingresa al servicio de Emergencias con cuadro de dolor pélvico crónico de 15 días de evolución localizado en región suprapúbica y en fosa ilíaca derecha, de alta intensidad, progresivo que atenúa levemente con Hioscina. Asocia disuria, fiebre cuantificada de $39^{\circ} \mathrm{C}$, náuseas y flujo vaginal no fétido, color café.

Es valorada inicialmente por el Servicio de Cirugía General.

Al examen físico se describe un abdomen blando, depresible, francamente doloroso a la palpación en FID y región suprapúbica.

Peristalsis presente. Signo de rebote negativo. Sin datos de irritación peritoneal. Al examen ginecológico se encuentran genitales externos sin lesiones. Especuloscopia que evidencia una leucorrea no grumosa, escasa, no fétida. Mucosa vaginal sin lesiones. Cérvix sano con hilos de DIU visibles. Tacto Vaginal: Levemente doloroso a la palpación bimanual con útero irregular, aumentado de tamaño con anexos libres. Movilización del cérvix no dolorosa.

Se le realizan múltiples exámenes de laboratorios entre ellos:
-
Examen general de orina: Sin datos de

- Hemograma completo

- Hemoglobina: $12.7 \mathrm{~g} / \mathrm{dL}$

- Leucocitos 12'000. Con diferencial de polimorfonucleares: $6.9 \%$

- Pruebas de función renal: Sin alteraciones.

- Electrolitos: Dentro de límites normales.

Se requiere valoración por Ginecología, quienes solicitan US pélvico, el cual reporta:

"Útero AVF de 80 x 43mm con 2 miomas corporales posteriores de $28 \times 11 \mathrm{~mm}$ y 18 x $17 \mathrm{~mm}$ respectivamente. Presencia de DIU localizado a $19 \mathrm{~mm}$ del fondo uterino. En FID presenta una colección hipoecoica heterogénea compatible con plastrón de 55 x 60 x $57 \mathrm{~mm}$ "

Es dada de alta por el servicio de Emergencias Ginecológicas para continuar control por Cirugía. Se le da tratamiento analgésico con Tramadol intravenoso y se coordina TAC de abdomen, el cual reporta:

"En fosa iliaca derecha se observa área poco definida con aumento difuso de densidad de la grasa peritoneal que mide aproximadamente $40 \mathrm{x}$ $7 \times 9.5 \mathrm{~mm}$ que se asocia a adenopatías de $22 \times$ $21 \mathrm{~mm}$, íleo distal con engrosamiento difuso de sus paredes. Proceso inflamatorio en FID que como primera posibilidad podría corresponder a un infarto omental."

La paciente es llevada a sala de operaciones. En la nota quirúrgica se describe:

"Laparotomía exploratoria donde se observa un "omental cake" así como un útero adherido a vejiga, intestino y parametrios, con múltiples lesiones palpables que impresionan miomas pero de imposible disección. No es posible visualizar anexos ni determinar origen de lesión. Se logra separar epiplón y se decide enviar a patología para determinar origen de la lesión y completar estudios."

La paciente es egresada del servicio de Cirugía con diagnóstico postquirúrgico: Observación por neoplasia uterina y Síndrome adherencial. Es referida a Ginecología para valoración de biopsia y seguimiento.

Es valorada en la consulta externa de Ginecología con un resultado de biopsia de epiplón: positivo por peritonitis aguda y crónica secundaria a Actinomicosis sp. 
Figura 1. Inflamación severa a base de neutrófilos polimorfonucleares, con zonas de abscedación, y granos de Actinomyces sp. (HE, 100x).

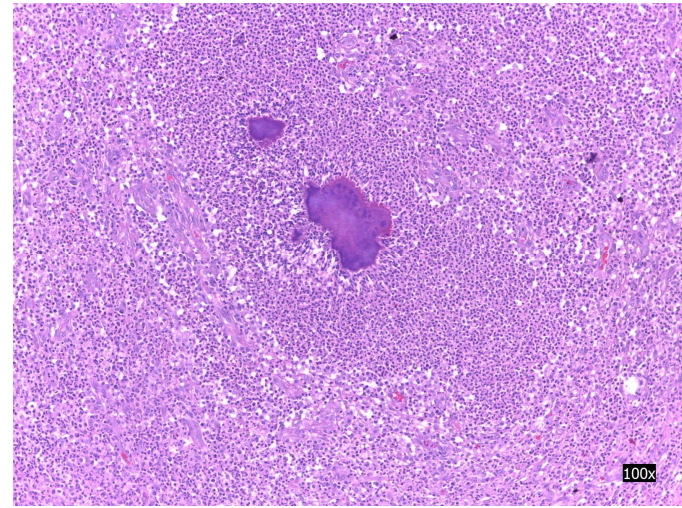

Fuente: Servicio de Patología. Hospital México.

Figura 2.Tinción de Grocottresalta los filamentos de Actinomyces sp. (Grocott, 400x).

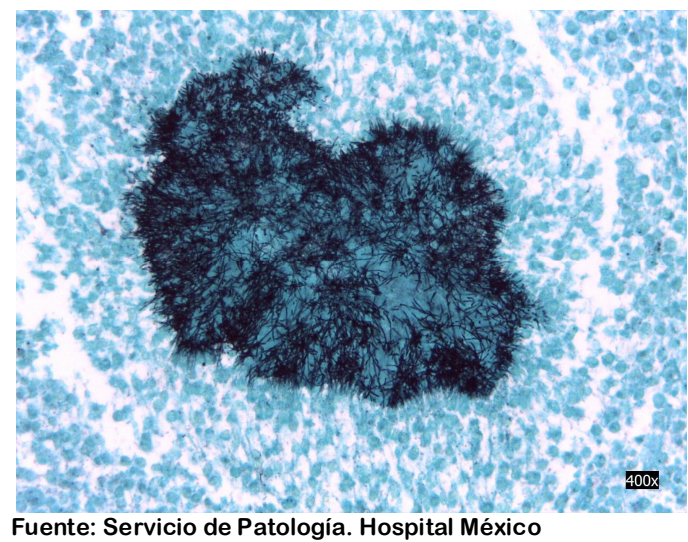

Es internada en el Servicio de Ginecología donde se retiró DIU, se interconsultó con Infectología para tratamiento antibiótico y manejo conjunto. Completó 1 mes de tratamiento con Penicilina intravenosa. Es egresada con tratamiento ATB oral (a completar 3 meses), TAC de abdomen control sin alteraciones y cita control en Ginecología.

\section{DISCUSIÓN Y ANÁLISIS DEL CASO}

La actinomicosis es una infección bacteriana producida por múltiples agentes etiológicos de poca actividad o avance lento que pertenecen al géneroActinomyces (bacterias grampositivas anaerobias o microaerófilas). El agente causal más común es el Actinomycesisraelii. Habita normalmente en la mucosa oral, faríngea, gastrointestinal (principalmente íleon terminal y ciego) y zona genital. La transgresión de la barrera mucosa es el mecanismo que da origen a la infección y en algunos casos puede desarrollar enfermedad grave ${ }^{(1,2)}$.

La infección por actinomices ha sido llamada "la gran imitadora” en la práctica clínica ${ }^{(3)}$. Produce un cuadro clínico ambiguo e inespecífico por lo que su diagnóstico es complejo.

Tres presentaciones iniciales que deben despertar la sospecha de esta infección son:

- La combinación de evolución crónica, la transgresión de fronteras hísticas y signos sugestivos de tumoración.

- Aparición de un conducto fistuloso que de manera espontánea muestra resolución pero reaparece.

- La infección refractaria o recidivante después de tratamiento breve ${ }^{(4)}$.

Presenta patología frecuentemente en la región faríngea $(50 \%)$, abdominal $(20 \%)$ y torácica (15\%). Debe existir un insulto o noxa que comprometa la integridad de la mucosa, por lo que la mayoría de los casos se desarrollan posterior a una apendicitis perforada, perforación intestinal, secundario a diverticulitis, presencia de cuerpo extraño en cavidad uterina como por ejemplo los dispositivos intrauterinos (DIU) o traumatismo ${ }^{(5)}$. El rasgo distintivo de la actinomicosis es su evolución crónica e indolente donde se presentan lesiones que pueden aparecer como induraciones individuales o múltiples ${ }^{(4)}$. Formación de abscesos, abundante granulación, tejido fibroso denso y formación de masas son algunas de las características producidas por el organismo posterior a la perforación de la mucosa ${ }^{(5)}$. Además presenta extensión a estructuras continuas mediante el cruce de límites anatómicos naturales y la formación de fístulas y trayectos sinuosos.

\section{Actinomicosis Pélvica}

Es una de las presentaciones más inusuales del Actinomyces, de difícil diagnóstico por su cuadro clínico y conocida por su relación con el uso de dispositivos intrauterinos e infección inflamatoria pélvica.

Es común que este organismo aparezca evidenciado en el Papanicolaou de rutina de mujeres con DIU ${ }^{(6,7,8)}$. El hallazgo del mismo no es predictor ni mucho menos diagnóstico de enferme- 
dad pélvica, dado que este es un organismo comensal del tracto genital femenino, por lo que se recomienda un manejo expectante de pacientes asintomáticas con esta manifestación ${ }^{(1)}$.

Las usuarias de dispositivos intrauterinos tienen una mayor posibilidad de desarrollar vaginosis bacteriana en comparación con la población en general ${ }^{(9)}$. El ambiente anaerobio desarrollado favorece el crecimiento del Actinomyces. La presencia de este organismo produce una citología más inflamatoria y una mayor frecuencia de infección mixta ${ }^{(10)}$. Ambas situaciones despliegan un mecanismo dual que perpetúa la presencia y sobrevivencia de este organismo en este grupo de pacientes.

Sin embargo, es poco probable que se logre detectar a las colonias de actinomyces en un estudio bacteriológico. ${ }^{(6)}$

Se reporta una incidencia menor del hallazgo de Actinomyces en el Papanicolaou de mujeres portadoras de DIU con levonorgestrel ${ }^{(7,8)}$. Además está comprobado que el riesgo de infección es directamente proporcional al tiempo de uso del DIU ${ }^{(3)}$.

El tratamiento de la actinomicosis pélvica depende de la extensión de la enfermedad y de la condición del paciente. La mayoría de los investigadores coinciden que las lesiones extensas deben tratarse quirúrgicamente con resección o drenaje, suplementado con una terapia antibiótica extensa. Penicilina $\mathrm{G}$ es el antibiótico de primera elección para actinomicosis ${ }^{(11)}$.

\section{CONCLUSIONES}

La actinomicosis pélvica es una presentación inusual del Actinomices, de difícil diagnóstico debido a su cuadro clínico inespecífico que puede enmascararse como patología neoplásica o incluso isquémica. El diagnóstico preoperatorio es difícil y desafortunadamente se retrasa con frecuencia ${ }^{(12)}$. El diagnóstico de actinomicosis casi nunca es considerado antes de que el patólogo lo establezca ${ }^{(4,6)}$

Actualmente no se han identificado factores de riesgo asociados al desarrollo de actinomicosis pélvica pero el tipo de DIU, la duración de su uso y las prácticas sexuales se han relacionado $(1,13)$
La paciente presentada anteriormente nos expone un caso estrechamente relacionado a la literatura:

- Nos exhibe una paciente con características que la hacen propensa a la infección por Actinomices: portadora de DIU por más de 5 años, sexualmente activa, PAP sin alteraciones.

- Se puede ejemplificar la complejidad del diagnóstico: desde patología quirúrgica isquémica, hasta posible neoplasia inclusive posterior a la intervención quirúrgica donde se evidencia la extensión a estructuras continuas mediante el cruce de límites anatómicos naturales.

- Se exponen los cambios histopatológicos de la lesión producida por este patógeno: Producción de abscesos, fístulas, abundante tejido de granulación, etc. Factores que dificultan precisar una etiológica.

- Se evidencia la importancia de los estudios de imagen para definir extensión de la lesión y posterior manejo, no así para un diagnóstico certero.

- Por último este caso clínico demuestra que el diagnóstico patológico acertado seguido de un tratamiento antibiótico adecuado son las mejores herramientas para un resultado satisfactorio, baja tasa de mortalidad y alta tasa de curación.

\section{BIBLIOGRAFÍA}

1.PieriniAngel L Nepote P Gianinetti L Wenger L. Actinomicosis Pélvica Presentación de Caso. Servicio de Cirugía General y Mixta del Hospital J.B. Iturraspe, Santa Fe, Argentina. Revista Argentina de Cirugía 2012.

2.Maxová K Menzlová E Kolařík D Dundr P Halaška M. Case Report: Pelvic Actinomycosis. Charles University in Prague. Karolinum Press, Prague 2012.

3.López- Olmos J Gasull J Vivar B. Actinomyces e infecciones mixtas en la citología cervicovaginal, en portadoras de DIU. ElsevierDoyma. Clínica e Investigación en Ginecología y Obstetricia 2010.

4.YeoJoo Kim JinaYoum JeeHyun Kim Byung Chul Jee. Actinomyces-likeorganisms in cervical smears: theassociationwithintrauterinedevice and pelvic inflammatory diseases. Obstetrics \& Gynecology Science 2014.

5.Hsi-Lin Hsiao Jung-TsungShen Hsi- ChihYeh Wen-Jeng $\mathrm{Wu}$ Chii-Jye Wang Chun- Hsiung Huang. Intra - and Extra Abdominal Actinomy- 
cosis mimicking Urachal Tumor in an Intrauterine Device Carrier: A Case Report. Kaohsiung J Med Sci January 2008. Vol 24. No 1. Elsevier. 6.Matsuda K Nakajima H N Khan K. et al. Preoperative diagnosis of pelvic actinomycosis by clinical cytology. Original Research. International Journal of Women's Health. 2012:a 527-533. 7.Alegría BJ González M Galleguillos M Whittle PC Franco S. C. Revisión de Infección Pelviana por Actinomices: Presentación de un Caso Clínico. Revista Chilena de Radiología. Vol. 9 No 4. 2003.

8.Merki-Feld GS Lebeda E Hogg B Keller PJ. The incidence of actinomyces-like organisms in Papanicolaoustained smears of copper- and levonorgestrel-releasing intrauterine devices. Contraception 2000; 61: 365 -8.

9.Barbone F Austin H Louv WC Alexander WJ. A followup study of methods of contraception, sexual activity, and rates of trichomoniasis, candidiasis, and bacterial vaginosis. Am J ObstetGynecol, 1990.

10.Mali B Joshi JV Wagle U et al. Actinomyces in cervical smears of women using intrauterine contraceptive devices. ActaCytol 1986

11.Yilmaz M Akbulut S Samdanci ET Yilmaz S. Abdominopelvic Actinomycosis Associated With an Intrauterine Device and Presenting With a Rectal Mass and Hydronephrosis: A Troublesome Condition for the Clinician. IntSurg 2012; 97: 254-259.

12.Vicedo Madrazo E.M Monje Beltrán ML Llobet Roma M MartÍn-Urda Diez-Canseco A Marqueta Sánchez JM. Caso Clínico. Actinomicosis pélvica en una paciente sin relaciones sexuales previas. ProgObstetGinecol. 2010;53(5):209-211

13.Bonnez W Lattimer G Mohanraj N Johnson T. Actinomycesnaeslundii as an Agent of Pelvic Actinomycosis in the Presence of an Intrauterine Device. Journal of Clinical Microbiology. Feb 1985, Vol 21, No 2. 273-275.

\section{FUENTES DE APOYO}

- Dr. Eduardo Alfaro Alcozer. Servicio de Patología. Hospital México.

\section{CONFLICTO DE INTERÉS}

El autor declara que en el presente reporte no hubo ningún conflicto de interés 